\title{
Analysis of clinical characteristics, nucleic acid detection and prognosis of 72 cases of common type Corona Virus Disease 2019(COVID-19) pneumonia in Hubei Province,China
}

Jiangrong Liao

Guizhou aerospace hospital

Yueyan Lou

Shanghai Jiao Tong University School of Medicine Affiliated Renji Hospital south campus

Shuoyan Lu

People' hospital of Songtao and Miao autonomous county

\section{Xueyun Xian}

Guizhou aerospace hospital

\section{Xiulin Wu}

The first affiliated hospital of army medical university

Daiwei Zhao

Ezhou Leishan hospital

\section{Zhuwen Luo}

Guizhou aerospace hospital

\section{Xueling Wu ( $\nabla$ wuxueling76@126.com )}

Shanghai Jiao Tong University School of Medicine Affiliated Renji Hospital

Research article

Keywords: COVID-19, Pneumonia, Clinical characteristics, Time of nucleic acid turning negative prognosis

Posted Date: June 3rd, 2020

DOl: https://doi.org/10.21203/rs.3.rs-29841/v1

License: (c) (i) This work is licensed under a Creative Commons Attribution 4.0 International License. Read Full License 


\section{Abstract}

Background: To describe the clinical characteristics and the time of nucleic acid turning negative in patients with common type Corona Virus Disease 2019(COVID-19) pneumonia and analyze the treatment time and prognostic factors.

Methods: It's a retrospective analysis of 72 cases of common type COVID-19 pneumonia diagnosed by nucleic acid test and Computed tomography (CT).

Results: The average time of nucleic acid test turning negative is $17.37 \pm 9.29$ days, and the average treatment time was $23.74 \pm 8.06$ days. There was no influence of Blood leukocyte count, Neutrophil count, lymphocyte count, C-reactive protein (CRP), glutamic-pyruvic transaminase (ALT), glutamic oxalacetic transaminase (AST), creatinine, D-dimer on the prognosis of the patients, whether univariate or multivariate logistic regression analysis. By $\chi 2$ test of the drugs and prognosis, we found that oseltamivir may have an impact on the prognosis of patients $(P<0.05)$, while other drugs have no significant impact on the prognosis of patients. Through the correlation analysis, we found that age, the admission temperature, leukocyte count, neutrophil count, CRP were positively correlated with the treatment time of the patients (both $\mathrm{P}<0.05$ ). Leukocyte count and neutrophil count were positively correlated with the time of nucleic acid test turning negative (both $\mathrm{P}<0.05$ ).

Conclusions: Age, the admission temperature, leukocyte count, neutrophil count, CRP were positively correlated with the treatment time of the patients. Leukocyte count and neutrophil count were positively correlated with the time of nucleic acid test turning negative. Oseltamivir may have an impact on the prognosis of patients, but the detailed role needs further study.

\section{Background}

Coronavirus belongs to the family of coronaviridae in the order Nidovirales. There are four groups of coronaviruses: alphacoronavirus infects mammals (human coronavirus NL63), betacoronavirus infects mammals (SARS CoV, MERS CoV, Bat CoV), gammacoronavirus infects birds (avian infectious bronchitis coronavirus), deltacoronavirus infects both birds and mammals (porcein delta CoV)[1]. The 2019 novel coronavirus pneumonia is caused by a novel betacoronavirus that is currently named 2019 novel coronavirus [2, 3]. The 2019 novel coronavirus is the seventh member of the envelope RNA coronavirus that infects human beings. Although most novel coronavirus infections are mild, the infection of coronavirus including 2019 new coronavirus, severe acute respiratory syndrome coronavirus (SARS-CoV) and Middle East respiratory syndrome coronavirus (MERS-CoV) may be serious or even fatal [1].

At present, there is no vaccine or specific antiviral therapy for COVID-19 that has been proved to be effective, so supportive therapy to reduce symptoms and protect multiple organ functions may be beneficial. 
In this study, we evaluated the clinical data, medication and prognosis of the patients who had been transferred and admitted to Leishan hospital,Ezhou, Hubei. We aimed to compare the demographic, clinical, laboratory, radiologic characteristics, drug and prognosis, time of nucleic acid test turning negative of patients with common type COVID-19 pneumonia.

\section{Methods}

\section{Study population}

This is a retrospective study, included 72 patients who diagnosed with COVID-19 pneumonia. They were confirmed with COVID-19 according to the Chinese guideline for diagnosis and treatment of COVID-19[4]. The patients were from Leishan hospital,Ezhou, Hubei. The patients were included from January 21, 2020 to February 25, 2020.

\section{Clinical Symptom Assessment And Test}

All patients were examined for body temperature at the time of admission, and collected the main complaints related to fever, cough, chest distress, fatigue, gastrointestinal discomfort, etc.

In order to determine SARS-CoV-2 infection, Nasopharyngeal swab samples were collected from all patients at the time of admission, and real-time reverse transcriptase polymerase chain reaction was used for detection according to the same protocol described previously[3], which were processed at the designated laboratory. These patients also underwent computer tomography (CT) scan after admission. 66 cases were confirmed by real-time reverse transcriptase polymerase chain reaction, 6 cases were negative or suspicious by real-time reverse transcriptase polymerase chain reaction test but suspected clinically.

Within 24 hours after admission, all patients underwent blood laboratory examination, including blood routine examination, CRP, liver and kidney function, blood lipid, blood coagulation, and other common pathogenic bacteria screening (influenza A, influenza B, tuberculosis, mycoplasma, chlamydia).

\section{Statistical analysis}

Continuous variables are expressed as median (IQR), $\mathrm{N}(\%)$ and mean \pm standard deviation $(\mathrm{M} \pm \mathrm{SD})$. Categorical values were expressed as frequencies or number (\%). $\chi 2$ test or the Fisher exact test is used to compare the classification data. Logistic regression analysis was used to analyze the influence of different laboratory tests and symptoms on the prognosis. Pearson correlation analysis was used to analyze the correlation between CT imaging, laboratory examination, time of nucleic acid test turning negative and treatment time. All analyses were performed with SPSS 25.0 (IBM SPSS) and Graphpad 6.0 software. A two-tailed $P$-value of $<0.05$ was considered statistically significant. 


\section{Results}

\section{Demographic and clinical characteristics}

A total of 72 novel coronavirus pneumonia patients were included in the study. The average age was $53.31 \pm 15.48$ years,there were 33 males $(45.8 \%)$ and 39 females( $54.2 \%) .10$ patients have contact history with Wuhan personnel $\mathbb{4}$ patients have contact history of non Wuhan pneumonia patients. None of the remaining patients had a clear history of epidemiological exposure. 66 cases were confirmed by real-time reverse transcriptase polymerase chain reaction, 6 cases were negative or suspicious by real-time reverse transcriptase polymerase chain reaction test but suspected clinically. In the past history of the patient, there were hypertension $(n=9[12.5 \%])$, diabetes $(n=6[8.0 \%])$, coronary heart disease $(n=3[4.0 \%])$ and chronic bronchitis $(n=3[4.0 \%])$. The main complaints were fever $(n=47[65.3 \%])$, cough $(n=39[54.2 \%])$, dyspnea $(n=18[25.0 \%])$, fatigue or myalgia $(n=19[26.4 \%])$ and gastrointestinal discomfort $(n=2[2.8 \%])$. These patients were also tested for influenza $A(n=3[4.0 \%])$, influenza $B(n=2[3.0 \%])$, tuberculosis $(n=$ $8[11.1 \%])$, mycoplasma $(n=18[25.0 \%])$,chlamydia $(n=7[10.0 \%])$. The average body temperature at the time of admission is $36.8 \pm 0.60 .70$ patients $(97.2 \%) \mathrm{T} \leq 38.0^{\circ} \mathrm{C}$ at the time of admission. According to the new guidelines for the diagnosis and treatment of coronavirus pneumonia, patients are divided into mild $(n=1[1.4 \%])$, common $(n=70[97.2 \%])$, severe $(n=1[1.4 \%])$ and critical $(0)$. There were 6 cases $(8.5 \%)$ with unilateral pulmonary infiltration and 65 cases $(91.5 \%)$ with bilateral pulmonary infiltration according to the $\mathrm{CT}$ imaging of the patients. We analyzed the correlation between CT imaging and prognosis, and found that bilateral or unilateral CT findings had no effect on the prognosis. The average time of nucleic acid test turning negative is $17.37 \pm 9.29$ days, and the average treatment time was $23.74 \pm 8.06$ days. All data are shown in Table 1. We conducted a multivariate logistic regression analysis of symptoms and prognosis, and found that the symptoms at the time of admission had no significant effect on the prognosis, as shown in Table 2. 
Table 1

Demographics and clinical characteristics of patients with common type COVID-19 pneumonia

\begin{tabular}{|ll|}
\hline & No(\%) \\
\hline Gender & \\
\hline Male & $33(45.8)$ \\
\hline Female & $39(54.2)$ \\
\hline Age (M \pm SD) & $53.31 \pm 15.48$ \\
\hline$\leq 65$ years & $57(79.2)$ \\
\hline$>65$ years & $15(20.8)$ \\
\hline Contact history & \\
\hline Contact history of Wuhan personnel & $10(13.9)$ \\
\hline Contact history of non Wuhan pneumonia patients & $4(5.6)$ \\
\hline Diagnostic approach & \\
\hline Real-time reverse transcriptase polymerase chain reaction & $66(91.7)$ \\
\hline Clinical diagnosis & $6(8.3)$ \\
\hline Past medical history & \\
\hline Hypertension & $9(12.5)$ \\
\hline Diabetes & $6(8.0)$ \\
\hline Coronary heart disease & $3(4.0)$ \\
\hline Chronic bronchitis & $3(4.0)$ \\
\hline Initial common symptoms & \\
\hline Fever & $39(65.3)$ \\
\hline Cough & $18(25.8)$ \\
\hline Dyspnea & \\
\hline Fatigue or myalgia & \\
\hline Upset gastro-intestinal & \\
\hline Virus infection & \\
\hline
\end{tabular}

a Correlation analysis of CT imaging and prognosis ( $\chi 2$ test)

Data are expressed as mean \pm standard deviation $(\mathrm{M} \pm \mathrm{SD})$ and $\mathrm{N}(\%)$ 


\begin{tabular}{|ll|}
\hline & No(\%) \\
\hline Influenza A & $3(4.0)$ \\
\hline Influenza B & $2(3.0)$ \\
\hline Tuberculosis & $8(11.1)$ \\
\hline Mycoplasma & $18(25.0)$ \\
\hline Chlamydia & $7(10.0)$ \\
\hline Temperature at admission $(\mathrm{M} \pm \mathrm{SD})$ & $36.8 \pm 0.60$ \\
\hline T $\leq 38.0^{\circ} \mathrm{C}$ & $70(97.2)$ \\
\hline T >38.0 ${ }^{\circ} \mathrm{C}$ & $2(2.8)$ \\
\hline Clinical classification & $1(1.4)$ \\
\hline Mild & $70(97.2)$ \\
\hline Common & $1(1.4)$ \\
\hline Severe & 0 \\
\hline Critical & \\
\hline Chest imaging, infiltrate ( $\left.{ }^{\mathrm{a}}=0.780\right)$ & $6(8.5)$ \\
\hline Unilateral & $65(91.5)$ \\
\hline Bilateral & $17.37 \pm 9.29$ \\
\hline Time of nucleic acid test turning negative (days) & $23.74 \pm 8.06$ \\
\hline Time of therapy (days) & \\
\hline a Correlation analysis of CT imaging and prognosis $(\mathrm{X} 2$ test $)$ \\
\hline Data are expressed as mean \pm standard deviation $(\mathrm{M} \pm \mathrm{SD})$ & $\mathrm{and} \mathrm{N( \% )}$ \\
\hline
\end{tabular}


Table 2

Logistic regression analysis of main complaints and prognosis of patients with common type of COVID-

19 pneumonia on admission

\begin{tabular}{|ll|}
\hline & $\mathbf{P}$ (multivariate logistic) \\
\hline Fever & 0.146 \\
\hline Cough & 0.343 \\
\hline Dyspnea & 0.513 \\
\hline Fatigue or myalgia & 0.153 \\
\hline Upset gastro-intestinal & 0.338 \\
\hline
\end{tabular}

\section{Laboratory Test}

The confirmed patients were examined for blood routine, liver and kidney function, blood coagulation indexes at the time of admission. We summarized these data in Table 3. The logistic regression analysis of Blood leukocyte count, Neutrophil count, lymphocyte count, C-reactive protein (CRP), glutamic-pyruvic transaminase (ALT), glutamic oxalacetic transaminase (AST), creatinine, D-dimer and prognosis was carried out. As shown in Table 4, there was no influence on the prognosis of the patients, whether univariate or multivariate logistic regression analysis. 
Table 3

Laboratory findings of patients with common type COVID-19

\begin{tabular}{|lll|}
\hline & M \pm SD & IQR \\
\hline Blood leukocyte count & $5.05 \pm 2.80$ & $3.57-5.59$ \\
\hline Neutrophil count & $3.23 \pm 2.71$ & $1.9-3.45$ \\
\hline Lymphocyte count & $1.42 \pm 0.46$ & $1.07-1.69$ \\
\hline CRP & $9.39 \pm 18.83$ & $0.26-6.53$ \\
\hline ALT & $34.52 \pm 24.88$ & $17.25-45.75$ \\
\hline AST & $29.47 \pm 18.26$ & $19.00-35.00$ \\
\hline Total cholesterol & $4.15 \pm 1.30$ & $3.21-4.97$ \\
\hline Triglyceride & $1.77 \pm 1.00$ & $1.08-2.13$ \\
\hline Creatinine & $66 \pm 18.49$ & $53.00-77.00$ \\
\hline Urea nitrogen & $3.82 \pm 1.16$ & $3.04-4.44$ \\
\hline D-dimer & $2.04 \pm 4.99$ & $0.38-1.49$ \\
\hline PT & $18.43 \pm 5.67$ & $17.00-18.70$ \\
\hline APTT & $28.87 \pm 5.18$ & $26.90-31.48$ \\
\hline Data are expressed as mean \pm standard deviation (M \pm SD) and median (IQR). \\
\hline Abbreviations: C-reactive protein (CRP), glutamic-pyruvic transaminase (ALT), glutamic oxalacetic \\
transaminase (AST), prothrombin time (PT), activated partial thromboplastin time (APTT).
\end{tabular}


Table 4

Logistic regression analysis of laboratory findings and prognosis of patients with common COVID-19 pneumonia

\begin{tabular}{|lll|}
\hline & $\mathbf{P}$ (univariate logistic) & $\mathbf{P}$ (multivariate logistic) \\
\hline Blood leukocyte count & 0.240 & 0.377 \\
\hline Neutrophil count & 0.293 & 0.474 \\
\hline Lymphocyte count & 0.888 & 0.357 \\
\hline CRP & 0.910 & 0.716 \\
\hline ALT & 0.420 & 0.678 \\
\hline AST & 0.588 & 0.770 \\
\hline Creatinine & 0.676 & 0.567 \\
\hline D-dimer & 0.571 & 0.674 \\
\hline $\begin{array}{l}\text { Abbreviations: C-reactive protein (CRP), glutamic-pyruvic transaminase (ALT), glutamic oxalacetic } \\
\text { transaminase (AST). }\end{array}$ \\
\hline
\end{tabular}

\section{Therapeutic Drug}

We summarized the drugs used in 72 patients during admission, as shown in Table 5. By $\chi 2$ test of these drugs and prognosis, we found that oseltamivir may have an impact on the prognosis of patients, while other drugs have no significant impact on the prognosis of patients. 
Table 5

Drugs used in the treatment of patients with common type COVID-19 pneumonia and their relationship with prognosis

\begin{tabular}{|llll|}
\hline Medicine & No $(\%)$ & X2 & P \\
\hline Arbidol & $52(72.2)$ & 0.527 & 0.468 \\
\hline Oseltamivir & $13(18.1)$ & 4.730 & 0.030 \\
\hline Wei lituo lopinavir ritonavir & $29(40.3)$ & 0.132 & 0.716 \\
\hline Ribavirin & $26(36.1)$ & 0.001 & 0.973 \\
\hline Interferon & $66(91.7)$ & 1.773 & 0.183 \\
\hline Human Immunoglobin & $10(13.9)$ & 0.054 & 0.816 \\
\hline Vitamin C & $16(22.2)$ & 0.829 & 0.363 \\
\hline Methylprednisolone & $2(2.8)$ & 0.610 & 0.435 \\
\hline Hydroxychloroquine & $1(1.4)$ & 0.295 & 0.587 \\
\hline Antibiotics & $19(26.4)$ & 1.125 & 0.289 \\
\hline * P<0.05 & & & \\
\hline $\begin{array}{l}\text { Data are expressed as } N(\%), \text { Pearson chi square }(\chi 2) \text { test for the relationship between drugs and } \\
\text { prognosis, a two-tailed } P \text {-value of }<0.05 \text { was considered statistically significant. }\end{array}$ & \\
\hline
\end{tabular}

\section{Influencing factors on treatment time and virus turning negative time}

Through the correlation analysis, we found that age, the admission temperature, leukocyte count, neutrophil count, CRP positively correlated with the treatment time of the patients, as shown in Fig. 1. The lymphocyte count, ALT, AST, Total cholesterol, Creatinine, D-dimer, prothrombin time (PT) and activated partial thromboplastin time (APTT) were not correlated with the treatment time of the patients. Leukocyte count and neutrophil count were positively correlated with the time of nucleic acid test turning negative (as shown in Fig. 2), while other indexes were not significantly correlated with the time of nucleic acid test turning negative.

\section{Discussion}

This study includes the basic clinical data, contact history, laboratory data, CT imaging, drug, time of nucleic acid test turning negative, time of therapy and prognosis. We found that most of these patients did not have a clear epidemiological contact history. It may be related to the increase of cases of COVID19 infection in other areas of Hubei Province besides Wuhan. In our study ,most of the patients had a 
admission temperature lower than $38^{\circ} \mathrm{C}$, which may be related to the fact that most of them were common patients. In previous studies, novel coronavirus pneumonia was mainly caused by low fever[5]. Some studies showed that elderly patients and severe patients were mainly hyperthermia[6, 7].

In this study, the CT imaging manifestations of the patients in hospital were mostly bilateral exudation. Studies have shown that novel coronavirus pneumonia is mainly manifested by bilateral exudation in СT[8]. We found that bilateral or unilateral CT findings had no effect on the prognosis. The average time of nucleic acid test turning negative is $17.37 \pm 9.29$ days. The average treatment time of patients is 23.74 \pm 8.06 days. To explore the correlation between CT imaging, pathology and viral load is helpful to judge the imaging features of prognosis and guide clinical treatment, which needs further study. Tao Al et al. Carried out a retrospective study on 601 patients in Wuhan who were positive for RT-PCR detection of new coronavirus, and found that the average time of positive initial detection turned negative was $6.9 \pm 2.3$ days[9]. However, different doctors may have different standards for the first nucleic acid test of nucleic acid positive patients, which may lead to different time for nucleic acid to turn negative in different hospitals. Some cases have been reported, including some of the patients in our study who turned negative after treatment and then positive after a period of time. Therefore, the frequency and time interval of detection need to be further explored. If the detection frequency is increased or the interval is prolonged, combined with the improvement of clinical symptoms, we can more accurately estimate the progress of the disease, and further determine whether the patient carries the virus or is infectious.

The main symptoms of the patient are fever, cough, fatigue, chest tightness and gastrointestinal discomfort, which are similar to novel coronavirus pneumonia reported elsewhere[6, 7, 10]. Logistic regression analysis of the main complaints and prognosis at the time of admission showed that although the incidence of fever, cough, chest distress, fatigue and gastrointestinal discomfort were different, the first symptom had no significant effect on the prognosis of the patients.

We conducted univariate and multivariate Logistic regression analysis on the influence of laboratory data on the prognosis of patients, and found that these laboratory data had no significant influence on the prognosis of patients. It may be related to the fact that the patients we included are basically ordinary patients. Some studies have shown that the elevated ALT, AST, CRP, prothrombin time, D-dimer, urea nitrogen, creatinine in the dead patients are higher than those in the convalescent patients[11]. However, through the correlation analysis of age, temperature at admission, laboratory data and the time of nucleic acid test turning negative and treatment time, we found that age, temperature at admission, leukocyte count, neutrophil count, C-reactive protein and treatment time were positively correlated. Leukocyte count and neutrophil count were positively correlated with the time of nucleic acid test turning negative.

If we take intervention measures as early as possible after finding the pathogen, it is the most effective to suppress the outbreak of the virus. However, the new coronavirus (nCov) has unknown sensitivity to treatment, is the main source of pandemic risk. Although in such an environment, the shallow global Pharmacopoeia of generic antiviral drugs limits the clinical effectiveness of treatment from the outbreak front, it can provide the most abundant information[12]. We analyzed the drugs used in all patients during 
hospitalization, as shown in the Table 5, the highest use rate of interferon and arbidol, but it seems that only oseltamivir has an impact on the prognosis of patients. But whether oseltamivir can improve the prognosis of patients needs further study to confirm. Previous studies have shown the potential effect of ribavirin on $\mathrm{nCoV}[13]$. Among the possible studies that should be completed are drug combinations that use the widely available abidol, interferon. The protease inhibitors lopinavir and ritonavir and oseltamivir neuraminidase are being studied. Remdesivir, which interferes with viral polymerase, can be tried[14]. Some open, multicenter RCT studies on therapeutic drugs are underway, which may provide important options for clinical treatment[15].

\section{Conclusion}

Most of the common type COVID-19 pneumonia patients had a admission temperature lower than $38^{\circ} \mathrm{C}$. Age, the admission temperature, leukocyte count, neutrophil count, CRP were positively correlated with the treatment time of the patients. Leukocyte count and neutrophil count were positively correlated with the time of nucleic acid test turning negative. Oseltamivir may have an impact on the prognosis of patients. For the detection time of whether the virus turns negative for the first time, and the effective therapeutic drugs of covid-19 need to be further studied.

\section{Limitations}

This study has several limitations. First of all, due to the limited medical resources, during this period only common type of COVID-19 pneumonia patients were hospitalized. Second, the study was conducted in a single central hospital with a limited sample size. As a result, the study may include a disproportionate number of patients with adverse outcomes. There may also be selection bias in determining the factors that affect clinical outcomes.

\section{Abbreviations}

Corona Virus Disease 2019(COVID-19), Computed tomography (CT), C-reactive protein (CRP), glutamicpyruvic transaminase (ALT), glutamic oxalacetic transaminase (AST), alphacoronavirus infects mammals (human coronavirus NL63), betacoronavirus infects mammals (SARS CoV, MERS CoV, Bat $\mathrm{CoV}$ ), deltacoronavirus infects both birds and mammals (porcein delta CoV), severe acute respiratory syndrome coronavirus (SARS-CoV), Middle East respiratory syndrome coronavirus (MERS-CoV), prothrombin time (PT), activated partial thromboplastin time (APTT).

\section{Declarations}

\section{Ethics approval and consent to participate}


Because this study uses a processed data set without any personal identification code, and does not involve any data set that may identify individuals, it does not need the approval of the ethics committee. Although there is no personal identity involved and no individual is harmed by the research, the research follows some guidelines to ensure the reliability of science.

\section{Consent for publication}

Not Applicable.

\section{Availability of data and materials}

The dataset used and/or analysed during the current study are available from the corresponding author on reasonable request.

\section{Competing interests}

The authors have declared no conflicts of interest.

\section{Funding}

There is no founding.

\section{Author Contributions:}

All authors were involved in drafting the article or revising it critically for important intellectual content, and all authors approved the final version to be submitted for publication. XW (Xueling Wu), DZ, ZL and XW (Xiulin Wu) had full access to all of the data in the study and take responsibility for the integrity of the data and the accuracy of the data analysis.

Study conception and design: JL, YL, XW (Xueling Wu).

Acquisition of the data: JL, SL, DZ.

Analysis and interpretation of the data: YL, XX, XW (Xiulin Wu), ZL.

\section{Acknowledgements}

Not Applicable. 


\section{References}

1. Zhu N, et al. A Novel Coronavirus from Patients with Pneumonia in China, 2019. N Engl J Med. 2020;382(8):727-33.

2. Lu R, et al. Genomic characterisation and epidemiology of 2019 novel coronavirus: implications for virus origins and receptor binding. Lancet. 2020;395(10224):565-74.

3. Huang C, et al. Clinical features of patients infected with 2019 novel coronavirus in Wuhan, China. Lancet. 2020;395(10223):497-506.

4. multicenter collaboration group of Department of, S., P. Technology of Guangdong, and p. Health Commission of Guangdong Province for chloroquine in the treatment of novel coronavirus, [Expert consensus on chloroquine phosphate for the treatment of novel coronavirus pneumonia]. Zhonghua Jie He He Hu Xi Za Zhi. 2020. 43(0): p. E019.

5. Xu T, et al., Clinical features and dynamics of viral load in imported and non-imported patients with COVID-19. Int J Infect Dis, 2020.

6. Lian J, et al., Analysis of Epidemiological and Clinical features in older patients with Corona Virus Disease 2019 (COVID-19) out of Wuhan. Clin Infect Dis, 2020.

7. Zhang G, et al. Analysis of clinical characteristics and laboratory findings of 95 cases of 2019 novel coronavirus pneumonia in Wuhan, China: a retrospective analysis. Respir Res. 2020;21(1):74.

8. Wu C, et al., Risk Factors Associated With Acute Respiratory Distress Syndrome and Death in Patients With Coronavirus Disease 2019 Pneumonia in Wuhan, China. JAMA Intern Med, 2020.

9. Ai T, et al., Correlation of Chest CT and RT-PCR Testing in Coronavirus Disease 2019 (COVID-19) in China: A Report of 1014 Cases. Radiology, 2020: p. 200642.

10. Xu XW, et al. Clinical findings in a group of patients infected with the 2019 novel coronavirus (SARSCov-2) outside of Wuhan, China: retrospective case series. BMJ. 2020;368:m606.

11. Chen T, et al. Clinical characteristics of 113 deceased patients with coronavirus disease 2019: retrospective study. BMJ. 2020;368:m1091.

12. Zhang H. Early lessons from the frontline of the 2019-nCoV outbreak. Lancet. 2020;395(10225):687.

13. Khalili JS, et al., Novel coronavirus treatment with ribavirin: Groundwork for evaluation concerning COVID-19. J Med Virol, 2020.

14. Wan Y, et al., Receptor Recognition by the Novel Coronavirus from Wuhan: an Analysis Based on Decade-Long Structural Studies of SARS Coronavirus. J Virol, 2020. 94(7).

15. Zhang Q, et al., Clinical trial analysis of 2019-nCoV therapy registered in China. J Med Virol, 2020.

\section{Figures}



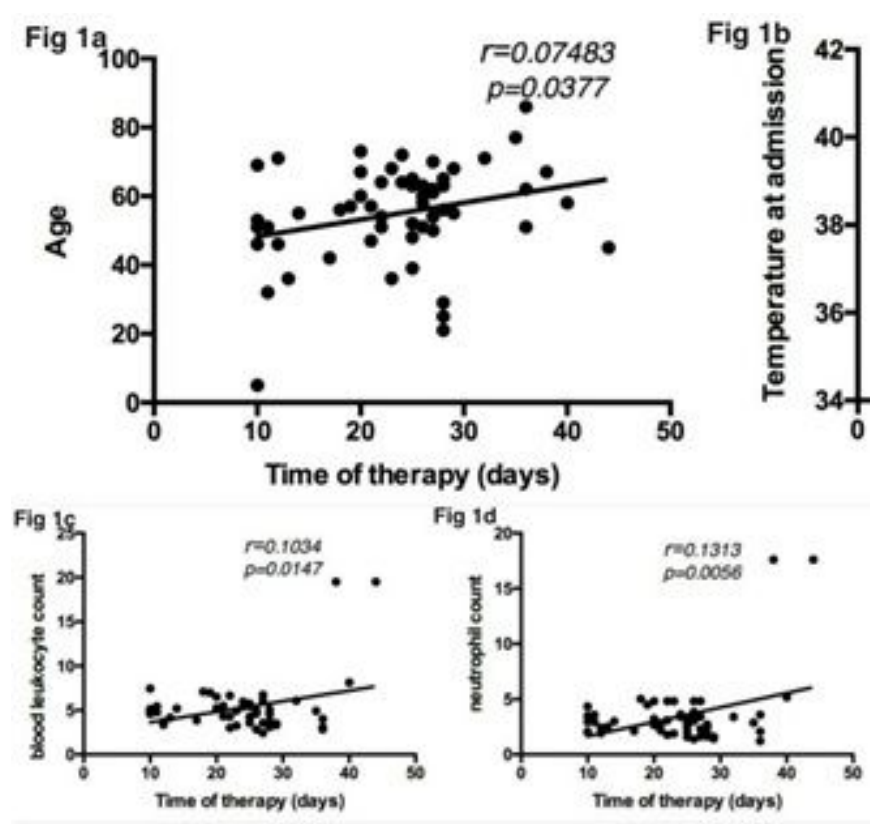

Fig 1b
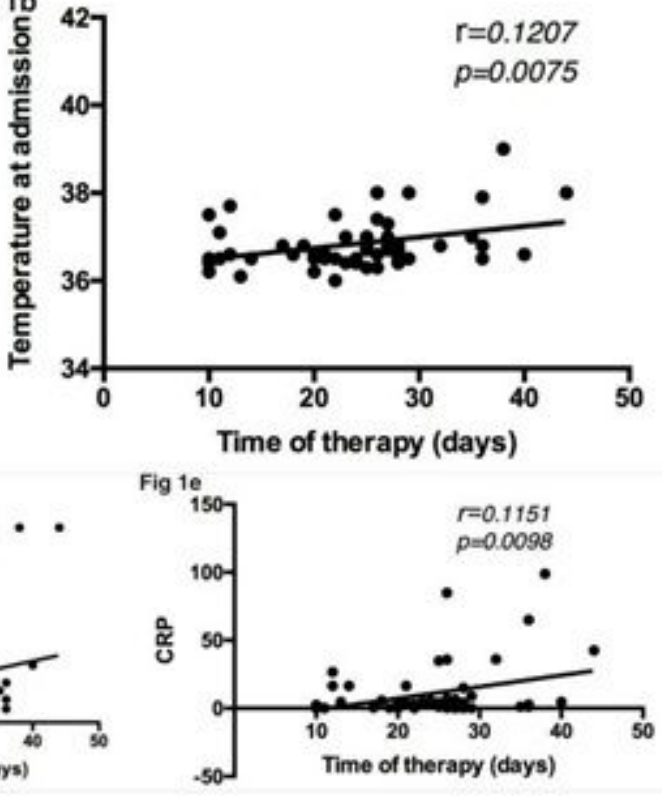

Figure 1

Correlation between age, temperature at admission, blood leukocyte count, Neutrophil count, CRP and time of therapy (days). The correlation analysis was performed to analyze the concentrations between time of therapy (days) and age (a), temperature at admission (b), blood leukocyte count (c), Neutrophil count (d), CRP (e) in patients with common type of COVID-19 pneumonia.

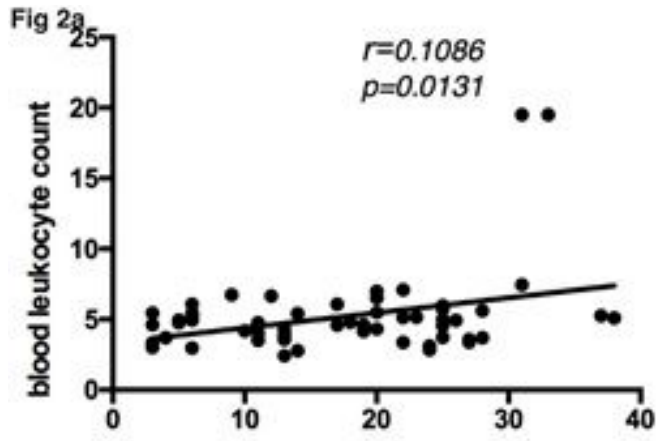

Time of nucleic acid test turning negative (days)

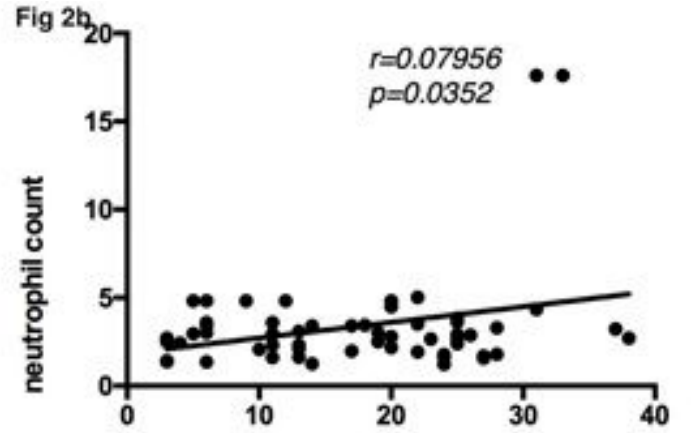

Time of nucleic acid test turning negative (days)

Figure 2

Correlation between blood leukocyte count, neutrophil count and time of nucleic acid test turning negative (days). The correlation analysis was performed to analyze the concentrations between time of nucleic acid test turning negative (days) and blood leukocyte count (a), neutrophil count (b) in patients with common type of COVID-19 pneumonia. 